\title{
Echelons of power series and Gabrielov's counterexample to nested linear Artin Approximation
}

\author{
M.E. ALONSO, F.J. CASTRO-JIMÉNEZ, H. HAUSER, C. KOUTSCHAN (1)
}

\begin{abstract}
Gabrielov's famous example for the failure of analytic Artin approximation in the presence of nested subring conditions is shown to be due to a growth phenomenon in standard basis computations for echelons, a generalization of the concept of ideals in power series rings.
\end{abstract}

\section{Introduction}

In the Séminaire Henri Cartan of 1960/61, Grothendieck posed the question whether analytically independent analytic functions are also formally independent $[\mathrm{Gr}] .{ }^{(2)}$ It came as a surprise when Gabrielov answered the question in 1971 in the negative. He constructed four analytic functions $e, f, g, h$ in three variables admitting one formal relation but no analytic one [Gb1]. To our knowledge, this is essentially the only known counterexample to Grothendieck's question. In an opposite direction, Pawłucki constructed analytic functions and a subset $Z$ of the reals for which there do exist analytic relations for parameter values outside $Z$ but there do not exist formal relations for parameters in $Z$ [Pa1]. In a later paper, Gabrielov gave a sufficient condition for a positive answer to Grothendieck's question in terms of the rank of the Jacobian matrix of the analytic functions [Gb2], see also [Pa2]. Much more generally, Popescu proved in 1985 a difficult approximation theorem which contains as a particular case a positive answer whenever the analytic functions are algebraic power series [Po1, Po2, Sp, Te]. Gabrielov's counterexample is based on an example of Osgood [Os] from 1916, complemented by a tricky construction and calculation. The deeper reason for the existence of formal divergent relations between analytically independent analytic functions remained mysterious over the years.

In this note we explain the genesis of the phenomenon in Gabrielov's example and provide a systematic way to construct many more counterexamples: It turns out that the existence of formal but not analytic relations is caused by accumulated growth occurrences in standard basis computations for echelons (an echelon is a generalization of an ideal in a power series ring, see below). Such a growth behaviour is well known for standard bases of ideals, but does not do any harm there due to the finiteness of the basis (which is ensured by the Noetherianity of the power series ring.) Standard bases of echelons need no longer be finite, and the iterated growth occurrence in their construction may indeed force divergence. We illustrate in the paper how this phenomenon is related to the presence of sufficiently fast converging coefficients of the (analytic) input series. In the example, the coefficients converge faster than exponentially.

For algebraic power series, the phenomenon does not happen. The echelon standard basis may still be infinite, but the convergence of the coefficients of the involved series seems to be sufficiently slow so as to ensure a positive answer to Grothendieck's question: whenever there is a formal linear relation respecting the scopes, there is also a convergent one (actually, even an algebraic one). The assertion for

Mathematics Subject Classification (2010): 32A05, 13F25, 13P10, 16W60, 14QXX, 14B12.

(1) This work was done in part during a Research-in-Teams program at the Erwin-Schrödinger Institute at Vienna, and the special semester on Artin approximation within the Chaire Jean Morlet at CIRM, Luminy-Marseille. M.E.A. was supported by MINECO MTM2014-55565 and UCM , IMI and Grupo 910444, F.J.C.-J. by MTM2013-40455-P and MTM2016- 75024-P, H.H. and C.K. by the Austrian Science Fund FWF, within the projects P-25652 and AI-0038211, respectively P29467-N32 and F5011-N15.

(2) Artin attributes in [Ar] Grothendieck's question to Abhyankar. 
algebraic series follows for instance from Popescu's approximation theorem (i.e., the fact that nested approximation holds for algebraic power series), whereas a direct explanation in terms of echelons is still lacking.

Our explanation of Gabrielov's example will be embedded in a short description of the division theorem for power series in the setting of echelons and the related notion of echelon standard basis. This is not mandatory to understand the example but should allow the reader to see its construction in a broader context.

\section{Gabrielov's example}

The first step towards Grothendieck's question, and this already appears in [Gb1], is to transcribe the existence of formal or analytic relations to a nested linear Artin approximation problem: Let $f_{1}(x), \ldots, f_{m}(x)$ be convergent power series in variables $x=\left(x_{1}, \ldots, x_{n}\right)$ and let $r\left(y_{1}, \ldots, y_{m}\right)$ be a (formal or analytic) relation between them, say,

$$
r\left(f_{1}(x), \ldots, f_{m}(x)\right)=0 .
$$

This is equivalent to saying that $r(y)$ belongs to the ideal of the formal, respectively convergent, power series ring $\mathbb{C}[[x, y]]$, respectively $\mathbb{C}\{x, y\}$, generated by the series $y_{i}-f_{i}(x)$, for $i=1, \ldots, m$. Therefore there exist power series $a_{1}(x, y), \ldots, a_{m}(x, y)$ such that

$$
r(y)=\sum_{i=1}^{m} a_{i}(x, y) \cdot\left(y_{i}-f_{i}(x)\right) .
$$

Here, the series $a_{i}$ are allowed to depend on both $x$ and $y$, whereas the series $r$ must be independent of $x$. This requirement is known in the context of Artin approximation as a "nested subring condition". Note that the unknown series $r$ and $a_{i}$ appear linearly in the equation. As an extension of Grothendieck's question one may then ask more generally whether linear nested Artin approximation holds for analytic functions: Given analytic functions $e$ and $f_{1}, \ldots, f_{m}$ in $n$ variables $x_{1}, \ldots, x_{n}$ such that the linear presentation

$$
e(x)=\sum_{i=1}^{m} \widehat{a}_{i}(x) \cdot f_{i}(x)
$$

holds with formal power series $\widehat{a}_{i}(x)$ depending only on the variables $x_{1}, \ldots, x_{s_{i}}$, for given $s_{i} \leq n$, does there exist a presentation

$$
e(x)=\sum_{i=1}^{m} a_{i}(x) \cdot f_{i}(x)
$$

with analytic functions $a_{i}(x)$ depending on the same sets of variables as $\widehat{a}_{i}(x)$ ?

Gabrielov also gives a counterexample to this case of linear nested analytic approximation: Consider the series $f=1, g=x \cdot\left(e^{z}-1\right)$, and $h=y z-x$ in three variables $x, y, z$. He then shows that the convergent series

$$
e(x, z)=\sum_{i=1}^{\infty} \sum_{j=0}^{\infty} \frac{i !}{(i+j) !} \cdot x^{i} z^{j+1}
$$

admits a presentation

$$
e=\widehat{a} \cdot f+\widehat{b} \cdot g+\widehat{c} \cdot h
$$

with formal series $\widehat{a}(x, y), \widehat{b}(x, y), \widehat{c}(x, y, z)$ but that there are no convergent series $a(x, y), b(x, y)$, $c(x, y, z)$ representing $e$ in this way. Setting

$$
I=\mathbb{C}\{x, y\} \cdot f+\mathbb{C}\{x, y\} \cdot g+\mathbb{C}\{x, y, z\} \cdot h
$$


with completion $\widehat{I}=\mathbb{C}[[x, y]] \cdot f+\mathbb{C}[[x, y]] \cdot g+\mathbb{C}[[x, y, z]] \cdot h$, one therefore has the strict inclusion of vector subspaces

$$
I \subsetneq \widehat{I} \cap \mathbb{C}\{x, y, z\} .
$$

We will investigate in this note the deeper reason behind this fact. To do so, we collect in the next section the basics about echelons, a generalization of the notion of ideals in power series rings. Subspaces as $I$ above are echelons, and their understanding is crucial for explaining Gabrielov's example. This explanation is presented in the section after the section on echelons.

\section{Echelons}

Let $x_{1}, \ldots, x_{n}$ be variables and $K[[x]]=K\left[\left[x_{1}, \ldots, x_{n}\right]\right]$ the ring of formal power series in $x_{1}, \ldots, x_{n}$ over a given field $K$. If $K$ is a valued field, we may also consider the subring $K\{x\}=K\left\{x_{1}, \ldots, x_{n}\right\}$ of convergent power series. The next definitions apply always equally to the convergent case. A finitary echelon is a $K$-subspace of $K[[x]]$ which can be written as a finite sum

$$
I=\sum_{i=1}^{k} K\left[\left[x_{1}, \ldots, x_{s_{i}}\right]\right] \cdot f_{i},
$$

with series $f_{i} \in K\left[\left[x_{1}, \ldots, x_{n}\right]\right]$ and integers $0 \leq s_{i} \leq n$, called the assigned scope of $f_{i}$. Sometimes, we also refer to the variables $x_{1}, \ldots, x_{s_{i}}$ themselves as the scope of $f_{i}$. Series $f_{1}, \ldots, f_{k}$ as above with assigned scopes $s_{1}, \ldots, s_{k}$ are called generators of $I$. When working with finitary echelons, we often tacitly assume that a generator system is already chosen. A linear combination $f=\sum_{i=1}^{k} a_{i} \cdot f_{i}$ is said to respect the scopes if $a_{i} \in K\left[\left[x_{1}, \ldots, x_{s_{i}}\right]\right]$ holds for all $i$. Each element $f$ of $I$ can be represented in this way. In certain situations, the sum $\sum_{i=1}^{k} K\left[\left[x_{1}, \ldots, x_{s_{i}}\right]\right] \cdot f_{i}$ will be direct, and then the presentation $f=\sum_{i=1}^{k} a_{i} \cdot f_{i}$ of elements $f \in I$ as a linear combination of $f_{1}, \ldots, f_{k}$ respecting the scopes is unique (compare this with the later analysis of Gabrielov's example where the involved echelon is indeed a direct sum).

One could also develop a concept of infinitely generated echelons, but this is not needed for the sequel, and will hence be omitted here.

For $f \in I$, we call $s(f)=\max \left\{s \in\{0, \ldots, n\}, K\left[\left[x_{1}, \ldots, x_{s}\right]\right] \cdot f \subset I\right\}$ the actual scope of $f$ in $I$. Clearly, the assigned scope $s$ of $f$ is less than or equal to the actual scope $s(f)$. In a theoretical context, we may always assign the actual scope to $f$, so that $s=s(f)$, and we then just speak of the scope of an element. But for actual computations and a given $f \in I$, it seems often impossible to determine the actual scope algorithmically, since it would require a constructive echelon membership test for the multiples of $f$. This aspect will play a role in Thm. 2, where only assigned scopes are considered.

The analogous definitions hold for $K$-subspaces of free modules $K[[x]]^{m}$ of the form

$$
I=\sum_{i=1}^{k} K\left[\left[x_{1}, \ldots, x_{s_{i}}\right]\right] \cdot f_{i} \subset K[[x]]^{m},
$$

with power series vectors $f_{i} \in K\left[\left[x_{1}, \ldots, x_{n}\right]\right]^{m}$. We call such subspaces finitary (module) echelons, with generators $f_{i}$ and assigned scopes $s_{i}$.

Let now $f_{1}, \ldots, f_{k}$ with scopes $s_{1}, \ldots, s_{k}$ be given generators of a finitary echelon $I \subset K[[x]]$ (or of a finitary module echelon $I \subset K[[x]]^{m}$ ). The (module) echelon of (linear) relations between $f_{1}, \ldots, f_{k}$ is the $K$-subspace

$$
\operatorname{Rel}\left(f_{1}, \ldots, f_{k}\right)=\left\{r \in \prod_{i=1}^{k} K\left[\left[x_{1}, \ldots, x_{s_{i}}\right]\right], \sum r_{i} f_{i}=0\right\}
$$

of $K[[x]]^{k}$ consisting of the linear relations between $f_{1}, \ldots, f_{k}$ respecting the scopes $s_{i}$. Here, we assign to a relation $r=\left(r_{1}, \ldots, r_{k}\right)$ the scope $t:=\min \left\{s_{i}, r_{i} \neq 0\right\}$, so that the inclusion $K\left[\left[x_{1}, \ldots, x_{t}\right]\right] \cdot r \subset$ $\operatorname{Rel}\left(f_{1}, \ldots, f_{k}\right)$ is ensured. 
Assume that a monomial order $<$ on $\mathbb{N}^{n}$ is chosen, i.e., a total ordering compatible with the addition in $\mathbb{N}^{n}$ and so that 0 is the smallest element. It induces an ordering, also denoted by $<$, on the set of monomials $x^{\alpha}$ of $K[[x]], \alpha \in \mathbb{N}^{n}$. For $f \in K[[x]]$, we denote by $\operatorname{in}(f)=\operatorname{in}(f)=x^{\alpha}$ the smallest monomial with respect to $<$ appearing in the expansion of $f$ (we always take the coefficient equal to 1 , and agree that $\operatorname{in}(0)=0$ ). It is called the initial monomial of $f$ with respect to $<$. For a finitary echelon $I$ in $K[[x]]$, denote by $\operatorname{in}(I)=\operatorname{in}(I)$ the associated initial echelon of $I$ : this is the $K$-subspace of $K[[x]]$ of power series whose expansion only involves monomials which are initial monomials in $(f)$ of elements $f$ of $I$. It is thus the $x$-adic closure of the subspace of $K[[x]]$ spanned by all initial monomials of elements of $I$. In general, in $(I)$ will not be a finitary echelon. For later use we restrict to monomial orders which admit no infinite bounded and strictly increasing sequences (thus, $\left(\mathbb{N}^{n},<\right)$ will be order equivalent to $\mathbb{N}$ with the usual order). We reserve the symbol (\#) for this condition; it would not hold for instance for a lexicographic monomial order on $\mathbb{N}^{n}$.

Assume that we are given generators $F=\left\{f_{1}, \ldots, f_{k}\right\}$ of $I$ with assigned scopes $s_{1}, \ldots, s_{k} \in$ $\{0, \ldots, n\}$,

$$
I=\sum_{i=1}^{k} K\left[\left[x_{1}, \ldots, x_{s_{i}}\right]\right] \cdot f_{i} .
$$

Our goal is to construct an echelon standard basis of $I$ from $f_{1}, \ldots, f_{k}$. This is a (possibly infinite) set of elements $g_{j}$ of $I, j \in \mathbb{N}$, with assigned scopes $t_{j}$, whose initial monomials $x^{\alpha_{j}}=\operatorname{in}\left(g_{j}\right)$ generate in $(I)$ topologically:

$$
\operatorname{in}(I)=\sum_{j \in \mathbb{N}}^{*} K\left[\left[x_{1}, \ldots, x_{t_{j}}\right]\right] \cdot x^{\alpha_{j}} .
$$

Here, the symbol $\sum^{*}$ stands for infinite sums of elements of the summands $K\left[\left[x_{1}, \ldots, x_{t_{j}}\right]\right] \cdot x^{\alpha_{j}}$, say, power series in $K[[x]]$ whose exponents belong to the set

$$
\bigcup_{j \in \mathbb{N}} \alpha_{j}+\left(\mathbb{N}^{t_{j}} \times 0^{n-t_{j}}\right) .
$$

Such sums converge in the $x$-adic topology of $K[[x]]$ since the total degree of the monomials $x^{\alpha_{j}}$ tends with $j$ towards infinity (here, we exclude wlog repetitions among these monomials). By "construction" we understand a possibly infinite algorithm, which "terminates" in the sense that it produces, for each initial monomial $x^{\alpha}$ of in $(I)$, in finitely many steps an element $f \in I$ together with an assigned scope $s$ such that $x^{\alpha} \in K\left[\left[x_{1}, \ldots, x_{s}\right]\right] \cdot \operatorname{in}(f)$. Our algorithm mimicks Buchberger's algorithm for the construction of Gröbner and/or standard bases of ideals of polynomials, respectively power series [Bu, GP]. We do not, however, divide the new elements after each step by the existing ones. The main difference to the case of ideals is that echelon standard bases are not necessarily finite sets of generators, so that the notion of "termination" of the algorithm has to be drafted properly. See Thm. 2 below for details. ${ }^{(3)}$

Denote by $x^{\alpha_{i}}$ the initial monomials of a finite set $F=\left\{f_{1}, \ldots, f_{k}\right\}$ of generators $f_{i}$ with scope $s_{i}$ of $I$. Set $A=\bigcup_{i=1}^{k} \alpha_{i}+\left(\mathbb{N}^{s_{i}} \times 0^{n-s_{i}}\right)$ and denote by $B=A^{c}=\mathbb{N}^{n} \backslash A$ its complement. Write $K[[x]]^{B}$ for the space of power series whose expansions involve only monomials with exponent in $B$. An echelon power series division of a series $f \in K[[x]]$ by $F$ with respect to $<$ is a decomposition

$$
f=\sum_{i=1}^{k} a_{i} f_{i}+b
$$

with quotients $a_{i} \in K\left[\left[x_{1}, \ldots, x_{s_{i}}\right]\right]$, remainder $b \in K[[x]]^{B}$, and so that

$$
\operatorname{in}(f-b)=\min \left\{\operatorname{in}\left(a_{i}\right) \cdot \operatorname{in}\left(f_{i}\right), i=1, \ldots, k\right\},
$$

(3) In the case of ideals of power series rings, standard bases are finite. If the ideals are generated by algebraic power series (and the coordinates are sufficiently generic), the construction of standard bases can be performed by a finite algorithm, see [ACH]. 
where the minimum refers to the ordering of the monomials of $K[[x]]$ induced by $<$. This is the analog requirement as for polynomial division in the case where the divisors, say ideal generators, are not yet (or not necessarily) a Gröbner basis. Note that in general the decomposition is not unique.

Theorem 1. (Division theorem for echelons) Let $F=\left\{f_{1}, \ldots, f_{k}\right\}$ be a finite set of series $f_{i}$ in $K\left[\left[x_{1}, \ldots, x_{n}\right]\right]$ with assigned scopes $0 \leq s_{i} \leq n$. Choose a monomial order $<$ on $\mathbb{N}^{n}$. For every series $f$ there exists an echelon power series division (with respect to $<$ )

$$
f=\sum_{i=1}^{k} a_{i} f_{i}+b
$$

of $f$ by $f_{1}, \ldots, f_{k}$ with respect to $<$.

Remarks. (a) If $f_{1}, \ldots, f_{k}$ form an echelon standard basis, the remainder $b$ of the division is unique (whereas the coefficients $a_{i}$ still need not be unique). Uniqueness of $b$ does not hold for arbitrary $f_{1}, \ldots, f_{k}$. Prescribing support conditions on the coefficients $a_{i}$ as in the proof below by choosing a partition $A=\dot{\cup} A_{i}$ of the set $A$ and requiring $\operatorname{supp}\left(a_{i} \cdot \operatorname{in}\left(f_{i}\right)\right) \subset A_{i}$ for all $i$, both the coefficients $a_{i}$ and the remainder $b$ can be made unique (though they will depend on the chosen partition of $A$ ).

(b) If $I$ is the echelon generated by $f_{1}, \ldots, f_{k}$ with scopes $s_{1}, \ldots, s_{k}$, and if we assume that also $f$ belongs to $I$ and has assigned scope $s$, there is a natural way to assign to the remainder $b$, which then again belongs to $I$, a scope: namely, define it as the minimum of $s$ and the scopes $s_{i}$ for those $i=1, \ldots, k$ for which $a_{i} \neq 0$. This value can either be maximized over all presentations $f=\sum_{i=1}^{k} a_{i} f_{i}+b$ (finding the maximum value may not be constructive), or it can be made unique by choosing a partition $A=\dot{\cup} A_{i}$ and support conditions on the $a_{i}$ so that the presentation is unique.

(c) We can check by Thm. 1 effectively whether an element $f$ belongs to $I$ up to degree $d$, since then we only need a finite part of the echelon standard basis, namely those elements whose initial monomials are not larger than all degree $d$ monomials.

(d) With a little more work (using elementary Banach space techniques), the division statement of the theorem can be established for convergent power series, cf. [HM, Thm. 5.1]. It does not hold in general for algebraic series.

(e) The division theorem can also be formulated for vectors of power series and finitary module echelons.

Proof. We shall show that the $K$-linear map

$$
\begin{gathered}
u: \prod_{i=1}^{k} K\left[\left[x_{1}, \ldots, x_{s_{i}}\right]\right] \times K[[x]]^{A^{c}} \rightarrow K[[x]], \\
\left(a_{1}, \ldots, a_{k}, b\right) \rightarrow \sum_{i=1}^{k} a_{i} f_{i}+b
\end{gathered}
$$

is surjective. Along the way, we shall in addition show that every series $f \in K[[x]]$ has a preimage $(a, b)$ so that the condition $\operatorname{in}(f-b)=\min \left\{\operatorname{in}\left(a_{i}\right) \cdot \operatorname{in}\left(f_{i}\right), i=1, \ldots, k\right\}$ holds.

Write $u=v+w$ where $v$ is the "monomial approximation" of $u$ given by the initial monomials $x^{\alpha_{i}}$ of $f_{1}, \ldots, f_{k}$, i.e., where $v$ is the linear map

$$
\begin{gathered}
v: \prod_{i=1}^{k} K\left[\left[x_{1}, \ldots, x_{s_{i}}\right]\right] \times K[[x]]^{A^{c}} \rightarrow K[[x]], \\
\left(a_{1}, \ldots, a_{k}, b\right) \rightarrow \sum_{i=1}^{k} a_{i} x^{\alpha_{i}}+b .
\end{gathered}
$$

By definition of $K[[x]]^{A^{c}}$, the map $v$ is surjective. We shall choose a linear subspace $N$ of the first factor $\prod_{i=1}^{k} K\left[\left[x_{1}, \ldots, x_{s_{i}}\right]\right]$ so that the restriction $v_{N}$ of $v$ to $N \times K[[x]]^{A^{c}}$ becomes an isomorphism of $K$-vectorspaces. Using the inverse of $v_{N}$ we shall then show that also the restriction $u_{N}$ of $u$ is an isomorphism. From this the surjectivity of $u$ follows. Our choice of $N$ will ensure in addition the requirement (*) in the decomposition $f=\sum_{i=1}^{k} a_{i} f_{i}+b$.

To construct $N$, we proceed as in the classical case of Gröbner or standard bases by defining a suitable partition of the set of exponents $A=\bigcup_{i=1}^{k} \alpha_{i}+\left(\mathbb{N}^{s_{i}} \times 0^{n-s_{i}}\right)$ [Gal, GP, HM]. There is no distinguished 
choice of the partition of $A$. Typically, one sets $A_{1}=\alpha_{1}+\left(\mathbb{N}^{s_{1}} \times 0^{n-s_{1}}\right)$, and then defines $A_{i}=$ $\left[\alpha_{i}+\left(\mathbb{N}^{s_{i}} \times 0^{n-s_{i}}\right)\right] \backslash \bigcup_{j<i} A_{j}$. Here its is advisable to order the elements $f_{1}, \ldots, f_{k}$ by decreasing scope, $s_{1} \geq \ldots \geq s_{k}$, in order to exhaust $A$ first by larger regions.

So let us fix a partition $A=\dot{\cup} A_{i}$ of the set $A$. Denote by $N_{i}=K[[x]]^{A_{i}} \subset K[[x]]$ the subspace of series with exponents in $A_{i}$, and set $N=\prod_{i=1}^{k} N_{i}$. It is then clear that the restriction $v_{N}$ of $v$ to $N \times K[[x]]^{A^{c}}$ is an isomorphism of $K$-vectorspaces. Let $v_{N}^{-1}$ be its inverse. We prove that the restriction $u_{N}$ of $u$ to $N \times K[[x]]^{A^{c}}$ is also an isomorphism. For this it is sufficient to show that the composition $u \circ v_{N}^{-1}=(v+w) \circ v_{N}^{-1}=\operatorname{Id}_{K[[x]]}+w \circ v_{N}^{-1}$ is an isomorphism of $K[[x]]$. The "formal inverse" $\sum_{i=0}^{\infty}\left(-w \circ v_{N}^{-1}\right)^{i}$ defines a linear map from $K[[x]]$ to $K[[x]]$ since applying $w \circ v_{N}^{-1}$ to a power series $h$ increases its initial monomial with respect to the ordering of the monomials induced by $<$. It is therefore the inverse to $u \circ v_{N}^{-1}$. This shows that $u_{N} \circ v_{N}^{-1}$ and hence also $u_{N}$ are isomorphisms. It follows that the map $u$ is surjective as claimed.

It remains to show $(*)$. We clearly have $\operatorname{in}(f-b) \geq \min \left\{\operatorname{in}\left(a_{i}\right) \cdot \operatorname{in}\left(f_{i}\right), i=1, \ldots, k\right\}$. If strict inequality would hold, the equality $f=\sum_{i=1}^{k} a_{i} f_{i}+b$ would imply, because of $b \in K[[x]]^{A^{c}}$, that $\operatorname{in}\left(a_{i}\right) \cdot \operatorname{in}\left(f_{i}\right)=\operatorname{in}\left(a_{j}\right) \cdot \operatorname{in}\left(f_{j}\right)$ for some pair $i \neq j$. This is impossible since $A_{i} \cap A_{j}=\emptyset$. The theorem is proven.

We have already mentioned that echelon standard bases of echelons need no longer be finite. However, the ideas of Buchberger's algorithm apply as well to construct the elements one by one. This goes as follows.

Theorem 2. (Echelon standard bases) Let $F=\left\{f_{1}, \ldots, f_{k}\right\}$ be a set of power series with assigned scopes $0 \leq s_{i} \leq n$ generating a finitary echelon $I$ in $K\left[\left[x_{1}, \ldots, x_{n}\right]\right]$,

$$
I=\sum_{i=1}^{k} K\left[\left[x_{1}, \ldots, x_{s_{i}}\right]\right] \cdot f_{i} .
$$

Fix a monomial order $<$ on $\mathbb{N}^{n}$ satisfying condition (\#). There exists an algorithm to enlarge $F$ iteratively so that, for every monomial $x^{\alpha}$ of the initial echelon in $(I)$ of $I$, one arrives after finitely many enlargements at a set $\widetilde{F}$ which contains an element $f$ of assigned scope $s$ in $I$ with $x^{\alpha} \in K\left[\left[x_{1}, \ldots, x_{s}\right]\right] \cdot \operatorname{in}(f)$.

Remarks. (a) Said differently, the algorithm produces in finitely many steps the elements of an echelon standard basis of $I$ up to any prescribed degree. An enlargement of $F$ is defined as a finite set $\widetilde{F}$ containing $F$ all whose elements belong again to $I$ and carry an assigned scope. In the proof, the algorithm for constructing these enlargements will be described explicitly.

(b) We do not pretend that the algorithm terminates in the sense that, in the construction of the echelon standard basis, after finitely many steps no more enlargements occur (and, in general, this will not happen). Moreover, even in the case where a finite echelon standard basis exists, the algorithm may produce infinitely many elements (most of which will be redundant). This is due to the fact that we do not apply division after each step.

Proof. We first explain the algorithm, and then show the required property. It is a variation of Buchberger's algorithm in the version of power series, with the additional requirement of respecting in each step the scopes of the involved elements.

Choose, for every pair $i \neq j$, the canonical minimal relation $\left(m_{i}, m_{j}\right) \in K[x]^{2}$ between the initial terms (i.e., initial monomials taken together with their coefficients) $e_{i} \cdot x^{\alpha_{i}}$ and $e_{j} \cdot x^{\alpha_{j}}$ of $f_{i}$ and $f_{j}$, where $e_{i}$ and $e_{j}$ denote the respective coefficients in $K$ and where $m_{i}$ and $m_{j}$ are terms with appropriate coefficients so that

$$
m_{i} \cdot e_{i} \cdot x^{\alpha_{i}}+m_{j} \cdot e_{j} \cdot x^{\alpha_{j}}=0
$$


It is clear that the relations are unique up to multiplication by constants in $K$. Set $S\left(f_{i}, f_{j}\right):=$ $m_{i} f_{i}+m_{j} f_{j}$. These linear combinations of $f_{i}$ and $f_{j}$ satisfy $\operatorname{in}\left(S\left(f_{i}, f_{j}\right)\right)>\operatorname{in}\left(m_{i} f_{i}\right)=\operatorname{in}\left(m_{j} f_{j}\right)$, i.e., there occurs a cancellation of (monomial multiples of) the initial monomials of $f_{i}$ and $f_{j}$.

In the algorithm, we will only consider linear combinations $g_{i j}:=S\left(f_{i}, f_{j}\right)$ for which both $m_{i} \in$ $K\left[x_{1}, \ldots, x_{s_{i}}\right]$ and $m_{j} \in K\left[x_{1}, \ldots, x_{s_{j}}\right]$ respect the assigned scopes of $f_{i}$ and $f_{j}$. The other $S\left(f_{i}, f_{j}\right)$ will be discarded. Observe here that if $m_{i}$ or $m_{j}$ violate the scope condition then all monomial relations between $e_{i} \cdot x^{\alpha_{i}}$ and $e_{j} \cdot x^{\alpha_{j}}$ violate it.

We assign to the elements $g_{i j}$ thus obtained the scope $s_{i j}:=\min \left\{s_{i}, s_{j}\right\}$ and add them to the set $F$. This will be done with all pairs $(i, j)$ satisfying the scope condition. The resulting set $\widetilde{F}$ together with the assigned scopes of its elements is considered as the first enlargement of $F$. We then iterate the procedure with $\widetilde{F}$. This completes the description of the algorithm. ${ }^{(4)}$

We now show that the algorithm fulfills the assertion of the theorem. Let $x^{\alpha}$ be a monomial of in $(I)$. We have to prove that, after finitely many enlargements of $F$, there is an element $f \in F$ with assigned scope $s$ in $I$ so that $x^{\alpha} \in K\left[\left[x_{1}, \ldots, x_{s}\right]\right] \cdot \operatorname{in}(f)$.

We may assume that we have already run the algorithm until arriving at a set $F=\left\{f_{1}, \ldots, f_{k}\right\}$ for which all subsequent new initial monomials appearing later in the algorithm are larger than $x^{\alpha}$. Indeed, $\operatorname{in}\left(g_{i j}\right)>\operatorname{in}\left(m_{i} f_{i}\right)=\operatorname{in}\left(m_{j} f_{j}\right)$ is strictly larger than the maximum of $\operatorname{in}\left(f_{i}\right)$ and in $\left(f_{j}\right)$. As we don't reconsider combinations $S\left(f_{i}, f_{j}\right)$ taken care of in earlier enlargements, it follows that the new initial monomials appearing after an enlargement are all larger than the minimum of the new initial monomials of the preceding enlargement. We conclude that the sequence of new initial monomials is unbounded. Hence, by hypothesis (\#) on the monomial order, the sequence must overtake $x^{a}$ eventually.

As $x^{\alpha} \in \operatorname{in}(I)$ we may write $x^{\alpha}=\operatorname{in}\left(\sum a_{i} f_{i}\right)$ for some $a_{i} \in K\left[\left[x_{1}, \ldots, x_{s_{i}}\right]\right]$ and $f_{i} \in F$. Set

$$
M:=\min \left\{\operatorname{in}\left(a_{i} \cdot f_{i}\right), i=1, \ldots, k\right\} .
$$

Clearly, $M \leq x^{\alpha}$. If $M=x^{\alpha}$ we are done: There is an $i$ so that $x^{\alpha}=M=\operatorname{in}\left(a_{i}\right) \cdot \operatorname{in}\left(f_{i}\right)$, hence $x^{\alpha} \in K\left[\left[x_{1}, \ldots, x_{s_{i}}\right]\right] \cdot \operatorname{in}\left(f_{i}\right)$.

If $M<x^{\alpha}$, we will see that the algorithm enlarges $F$ to a set

$$
\widetilde{F}=\left\{f_{1}, \ldots, f_{k}, f_{k+1}, \ldots, f_{\tilde{k}}\right\}
$$

with assigned scopes $s_{i}$ for $f_{i}$, and we then construct a presentation $f=\sum_{i=1}^{\tilde{k}} \widetilde{a}_{i} f_{i}$ of $f$ with coefficients $\widetilde{a}_{i} \in K\left[\left[x_{1}, \ldots, x_{s_{i}}\right]\right]$ so that

$$
\widetilde{M}:=\min \left\{\operatorname{in}\left(\widetilde{a}_{i} \cdot f_{i}\right), i=1, \ldots, \tilde{k}\right\}>\min \left\{\operatorname{in}\left(a_{i} \cdot f_{i}\right), i=1, \ldots, k\right\}=M .
$$

This procedure is then repeated. By hypothesis (\#) on the monomial order, the resulting strictly increasing sequence of monomials $M, \widetilde{M}, \ldots$ must reach $x^{\alpha}$ after finitely many iterations. That is what we want to prove.

To do so, let $C$ be the set of indices $i$ with $\operatorname{in}\left(a_{i}\right) \cdot \operatorname{in}\left(f_{i}\right)=M$. We necessarily have $|C| \geq 2$, since, due to the inequality

$$
\operatorname{in}\left(\sum_{i=1}^{k} a_{i} f_{i}\right)>\min \left\{\operatorname{in}\left(a_{i} \cdot f_{i}\right), i=1, \ldots, k\right\},
$$

a cancellation of (monomial multiples of) initial monomials $\operatorname{in}\left(f_{i}\right)=x^{\alpha_{i}}$ must occur in the sum $\sum_{i=1}^{k} a_{i} f_{i}$. Let $c_{i}$ and $e_{i}$ in $K$ denote the coefficients of the monomials $\operatorname{in}\left(a_{i}\right)$, respectively $\operatorname{in}\left(f_{i}\right)$, of $a_{i}$, respectively $f_{i}$. It follows that the vector $r \in K[x]^{k}$ with entries $r_{i}=c_{i} \cdot \operatorname{in}\left(a_{i}\right)$ if $i \in C$ and

(4) Observe here that in the subsequent enlargements one does not need to reconsider combinations $S\left(f_{i}, f_{j}\right)$ of elements $f_{i}, f_{j}$ which have been taken care of earlier. 
$r_{i}=0$ otherwise forms a monomial relation in $\prod_{i=1}^{k} K\left[x_{1}, \ldots, x_{s_{i}}\right]$ between the terms $e_{i} \cdot \operatorname{in}\left(f_{i}\right)$, for $i=1, \ldots, k$,

$$
\sum_{i=1}^{k} r_{i} \cdot e_{i} \cdot \operatorname{in}\left(f_{i}\right)=\sum_{i \in C} c_{i} e_{i} \cdot \operatorname{in}\left(a_{i} \cdot f_{i}\right)=\left(\sum_{i \in C} c_{i} e_{i}\right) \cdot M=0 .
$$

For each pair $j \neq \ell$ in $C$, denote by $m_{j \ell} \in K[x]^{k}$ the relation vector between the monomials $e_{i} \cdot \operatorname{in}\left(f_{i}\right)$, $i=1, \ldots, k$, whose only non-zero entries occur for indices $j$ and $\ell$ and are the terms $m_{j}$ and $m_{\ell}$ appearing in the minimal monomial relation $m_{j} \cdot e_{j} \cdot x^{\alpha_{j}}+m_{\ell} \cdot e_{\ell} \cdot x^{\alpha_{\ell}}=0$ defined earlier in the description of the algorithm,

$$
m_{j \ell}=\left(0, \ldots, 0, m_{j}, 0, \ldots, 0, m_{\ell}, 0, \ldots, 0\right) \in K[x]^{k} .
$$

In order not to have to exclude the case $j=\ell$ we may set all $m_{j j}$ equal to 0 . We leave it as a (simple) combinatorial exercise to check that the vectors $m_{j \ell}^{\prime} \in K[x]^{|C|}$ obtained from $m_{j \ell}$ by taking only the components with index in $C$ form a generator system of the module echelon of relations between the terms $e_{i} \cdot \operatorname{in}\left(f_{i}\right)$, for $i \in C$, respecting the scopes.

The entry of the vector $r$ at index $i$ belongs to $K\left[x_{1}, \ldots, x_{s_{i}}\right]$, by the choice of $a_{i}$ in $K\left[\left[x_{1}, \ldots, x_{s_{i}}\right]\right]$, and the same holds for $m_{i} \in K\left[x_{1}, \ldots, x_{s_{i}}\right]$. Now notice that if we have a sum $h=\sum h_{i}$ with in $\left(h_{i}\right)=M$ for all $i$ and so that in $(h)>M$ then $h=\sum \lambda_{j \ell} \cdot S\left(h_{j}, h_{\ell}\right)$ for some $\lambda_{j \ell} \in K$. Furthermore, we have $\operatorname{in}\left(S\left(h_{j}, h_{\ell}\right)\right)>M$ for all $j, \ell$. In view of this we may therefore write

$$
r=\sum_{j, \ell \in C} b_{j \ell} \cdot m_{j \ell},
$$

for some coefficients $b_{j \ell}$ which are monomials in $K\left[x_{1}, \ldots, x_{s_{j \ell}}\right]$, with $s_{j \ell}=\min \left\{s_{j}, s_{\ell}\right\}$ as above.

In the description of the algorithm we defined elements $g_{j \ell}=m_{j \ell} \cdot\left(f_{1}, \ldots, f_{k}\right)=m_{j} f_{j}+m_{\ell} f_{\ell}$ with assigned scope $s_{j \ell}=\min \left\{s_{j}, s_{\ell}\right\}$ (the dot represents the scalar product in $K[[x]]^{k}$ ). We enlarge now $F$ to a set $\widetilde{F}$ by adding all $g_{j \ell}$, for $j, \ell \in C$. Denote by $f_{j \ell}=g_{j \ell} \in \widetilde{F}$ these new elements, and assign to them the scopes $s_{j \ell}:=\min \left\{s_{j}, s_{\ell}\right\}$. We get

$$
\sum_{i=1}^{k} r_{i} f_{i}=\sum_{j, \ell \in C} b_{j \ell}\left(m_{j} f_{j}+m_{\ell} f_{\ell}\right)=\sum_{j, \ell \in C} b_{j \ell} g_{j \ell}=\sum_{j, \ell \in C} b_{j \ell} f_{j \ell} .
$$

Decompose all $a_{i}$ into $a_{i}=c_{i} \cdot \operatorname{in}\left(a_{i}\right)+a_{i}^{\prime}$ for some $a_{i}^{\prime} \in K\left[\left[x_{1}, \ldots, x_{s_{i}}\right]\right]$ with in $\left(a_{i}^{\prime}\right)>\operatorname{in}\left(a_{i}\right)$. Then

$$
\begin{aligned}
f & =\sum_{i=1}^{k} a_{i} f_{i}=\sum_{i \notin C} a_{i} f_{i}+\sum_{i \in C}\left(c_{i} \cdot \operatorname{in}\left(a_{i}\right)+a_{i}^{\prime}\right) f_{i} \\
& =\sum_{i \notin C} a_{i} f_{i}+\sum_{i \in C} a_{i}^{\prime} f_{i}+\sum_{i=1}^{k} r_{i} f_{i} \\
& =\sum_{i \notin C} a_{i} f_{i}+\sum_{i \in C} a_{i}^{\prime} f_{i}+\sum_{j, \ell \in C} b_{j \ell} f_{j \ell} \\
& =: \sum_{i=1}^{k} \widetilde{a}_{i} f_{i}+\sum_{j, \ell \in C} \widetilde{a}_{j \ell} f_{j \ell},
\end{aligned}
$$

with respective coefficients $\widetilde{a}_{i} \in K\left[\left[x_{1}, \ldots, x_{s_{i}}\right]\right]$ and $\widetilde{a}_{j \ell} \in K\left[\left[x_{1}, \ldots, x_{s_{j \ell}}\right]\right]$, where $\widetilde{a}_{i}:=a_{i}$ for $i \notin C$ and $\widetilde{a}_{i}:=a_{i}^{\prime}$ for $i \in C$, and where $\widetilde{a}_{j \ell}:=b_{j \ell}$. This is a new presentation of $f$ as a linear combination of elements of our enlarged set $\widetilde{F}$. The scopes are respected. The first summand in the last line satisfies by definition of $C$ and $a_{i}^{\prime}$ the inequality

$$
\min \left\{\operatorname{in}\left(\widetilde{a}_{i}\right) \cdot \operatorname{in}\left(f_{i}\right), i=1, \ldots, k\right\}>M=\min \left\{\operatorname{in}\left(a_{i}\right) \cdot \operatorname{in}\left(f_{i}\right), i=1, \ldots, k\right\} .
$$

As for the second summand, recall that $\operatorname{in}\left(f_{j \ell}\right)=\operatorname{in}\left(g_{j \ell}\right)>\operatorname{in}\left(m_{j} f_{j}\right)=\operatorname{in}\left(m_{\ell} f_{\ell}\right)$ and $\operatorname{in}\left(m_{j}\right)=\operatorname{in}\left(a_{j}\right)$ for all $j, \ell \in C$. This implies that also

$$
\min \left\{\operatorname{in}\left(\widetilde{a}_{j \ell}\right) \cdot \operatorname{in}\left(f_{j \ell}\right), j, \ell \in C\right\}>M .
$$

We have found, after the enlargement of $F$ to $\widetilde{F}$, a presentation

$$
f=\sum_{i=1}^{k} \widetilde{a}_{i} f_{i}+\sum_{j, \ell \in C} \widetilde{a}_{j \ell} f_{j \ell}
$$

of $f$ as a linear combination respecting the scopes of the elements of $\widetilde{F}$ and with larger value 


$$
\widetilde{M}:=\min \left\{\operatorname{in}\left(\widetilde{a}_{i}\right) \cdot \operatorname{in}\left(f_{i}\right), \operatorname{in}\left(\widetilde{a}_{j \ell}\right) \cdot \operatorname{in}\left(f_{j \ell}\right) ; i=1, \ldots, k ; j, \ell \in C\right\} .
$$

Repeating the construction we produce by successive enlargements of $F$ a sequence of monomials $M<\widetilde{M}<\ldots$ which eventually attains $x^{\alpha}$. This is what had to be shown.

\section{Pseudo-code of algorithm of Theorem 2}

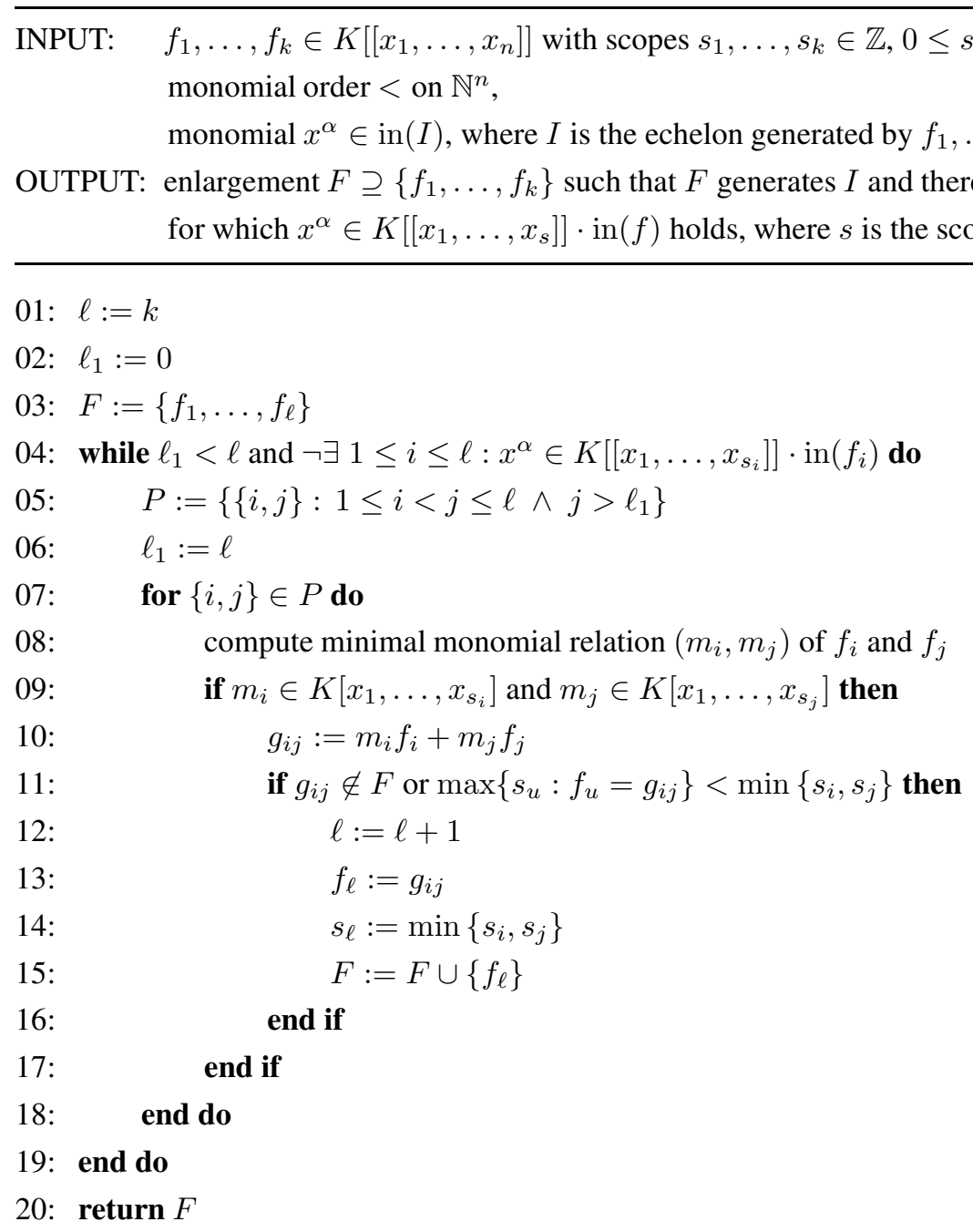

This algorithm, although it follows closely Buchberger's algorithm, does not reduce the S-polynomials. If we wanted to include division with remainder into the algorithm, its presentation would become much more complicated, which is related to the determination of the scope of newly added elements. Clearly, the scope of the new element should be the minimum of all scopes of elements that were used in the division. But then, we have to record also intermediate elements in the reduction with maximal possible scope. We illustrate the problem with an example: assume that $f_{1}, f_{2}, f_{3}$ have the scopes $s_{1}=s_{2}=2$, and $s_{3}=1$. Then we assign to $g_{1,2}=m_{1} f_{1}+m_{2} f_{2}$ the scope 2 , but after reducing it with $f_{3}$, we have to assign scope 1 . If we only add the final result (with scope 1 ) to $F$, we may hence miss an element of the standard basis of $I$. For actual computations, this conceptual version of the algorithm may be very inefficient, and therefore, in the next section, we will apply division with remainder to the S-polynomials, since the above-mentioned problem does not occur there.

\section{Analysis of Gabrielov's example}

We now return to the study of Gabrielov's example, where $f=1$ and $g=x \cdot\left(e^{z}-1\right)$ have assigned scope $x, y$, and where $h=y z-x$ has assigned scope $x, y, z$ (for clarity, we indicate instead of the value 
of the scope of the generators those variables which are allowed to appear in the series with which the generators are multiplied). As mentioned in the introduction, the explanation of the example does not require theorems 1 and 2, though these results help to put things in the right perspective.

Note first that the sums in our chosen echelons $I=\mathbb{C}\{x, y\} \cdot f+\mathbb{C}\{x, y\} \cdot g+\mathbb{C}\{x, y, z\} \cdot h$ and $\widehat{I}=\mathbb{C}[[x, y]] \cdot f+\mathbb{C}[[x, y]] \cdot g+\mathbb{C}[[x, y, z]] \cdot h$ are direct: If we had a non-trivial linear relation

$$
a(x, y) \cdot 1+b(x, y) \cdot x \cdot\left(e^{z}-1\right)+c(x, y, z) \cdot(y z-x)=0,
$$

setting $z=\frac{x}{y}$ would express $e^{\frac{x}{y}}$ as a quotient of power series in $x$ and $y$, which is impossible. Now order the monomials $x^{i} y^{j} z^{k}$ lexicographically by their exponents so that $z<_{\text {lex }} y<_{\text {lex }} x$. This order does not satisfy condition (\#) from above, since it allows bounded infinite strictly increasing sequences. This violation of (\#) does not alter the explanation of the example, and as the choice of $<_{\text {lex }}$ simplifies the presentation, we admit it here as an appropriate order.

The initial monomials of $f, g$ and $h$ with respect to $<_{l e x}$ are in $(f)=1, \operatorname{in}(g)=x z$, and $\operatorname{in}(h)=y z$. A lengthy check shows that the initial echelon in $(I)$ of $I$ equals

$$
\operatorname{in}(I)=\mathbb{C}\{x, y\} \cdot 1+\left(\sum_{k=1}^{\infty} \mathbb{C}\{x\} \cdot x^{k} z^{k}\right) \cap \mathbb{C}\{x, y, z\}+\mathbb{C}\{x, y, z\} \cdot y z .
$$

The sum $\sum_{k=1}^{\infty} \mathbb{C}\{x\} \cdot x^{k} z^{k}$ is well defined as a subspace of $\mathbb{C}[[x, y, z]]$, since the degree of the summands tends to infinity. So we may take its intersection with $\mathbb{C}\{x, y, z\}$. Similarly, we have

$$
\operatorname{in}(\widehat{I})=\mathbb{C}[[x, y]] \cdot 1+\sum_{k=1}^{\infty} \mathbb{C}[[x]] \cdot x^{k} z^{k}+\mathbb{C}[[x, y, z]] \cdot y z .
$$

Both subspaces are no longer finitary echelons since they require infinitely many "generators". We will not use these decompositions of in $(I)$ and $\operatorname{in}(\widehat{I})$ in the sequel, but it is helpful to keep them in mind.

We now start the algorithm for the construction of the echelon standard basis of $I$. For our purposes, it will be convenient to take some shortcuts using Thm. 1 by dividing new elements by the preceding ones, so as to simplify the resulting series. Moreover, we will not show that the constructions produce eventually all initial monomials of $I$. In this sense, the analysis of the example relies on a slightly modified version of the algorithm of Thm. 2.

Recall that $f, g$ and $h$ have initial monomials $1, x z$ and $y z$. As $\mathbb{C}[[x, y]] \cdot 1 \cap \mathbb{C}[[x, y]] \cdot x z=0$ and $\mathbb{C}[[x, y]] \cdot 1 \cap \mathbb{C}[[x, y, z]] \cdot y z=0$, we can only take one linear combination, say, of $g$ and $h$, namely $S(g, h)=-y \cdot g+x \cdot h$. Division of this series by $f, g$ and $h$ with respect to the assigned scopes yields the element

$$
\begin{aligned}
g_{2} & =-y \cdot g+x \cdot h+\left[x^{2} \cdot f+\frac{1}{2} x \cdot g+z^{-1} x \cdot\left(e^{z}-1-z\right) \cdot h\right] \\
& =x^{2} \cdot f-\left(y-\frac{1}{2} x\right) \cdot g+z^{-1} x \cdot\left(e^{z}-1\right) \cdot h \\
& =\frac{1}{2} \cdot z^{-1} x^{2} \cdot\left(e^{z} \cdot(z-2)+z+2\right) \\
& =\frac{1}{2} \cdot x^{2} \cdot \sum_{k=2}^{\infty} \frac{k-1}{(k+1) !} \cdot z^{k} \\
& =\frac{1}{12} \cdot\left[x^{2} z^{2}+\frac{1}{2} \cdot x^{2} z^{3}+\frac{3}{20} \cdot x^{2} z^{4}+\frac{1}{30} \cdot x^{2} z^{5}+\frac{1}{168} \cdot x^{2} z^{6}+\frac{1}{1120} \cdot x^{2} z^{7}+\ldots\right] .
\end{aligned}
$$

It has assigned scope $x, y$, and initial monomial

$$
\operatorname{in}\left(g_{2}\right)=x^{2} z^{2}
$$

This monomial does not belong to $\mathbb{C}[[x, y]] \cdot 1+\mathbb{C}[[x, y]] \cdot x z+\mathbb{C}[[x, y, z]] \cdot y z$. So we have found a new initial monomial of in $(\widehat{I})$. We iterate the process of taking linear combinations and then reducing by division. The first few elements one obtains after $f, g, h$ and $g_{2}$ are 


$$
\begin{aligned}
& g_{3}=\frac{1}{720} \cdot\left[x^{3} z^{3}+\frac{1}{2} \cdot x^{3} z^{4}+\frac{1}{7} \cdot x^{3} z^{5}+\frac{5}{168} \cdot x^{3} z^{6}+\frac{5}{1008} \cdot x^{3} z^{7}+\ldots\right] \\
& g_{4}=\frac{1}{100800} \cdot\left[x^{4} z^{4}+\frac{1}{2} \cdot x^{4} z^{5}+\frac{5}{36} \cdot x^{4} z^{6}+\frac{1}{36} \cdot x^{4} z^{7}+\ldots\right] \\
& g_{5}=\frac{1}{25401600} \cdot\left[x^{5} z^{5}+\frac{1}{2} \cdot x^{5} z^{6}+\frac{3}{22} \cdot x^{5} z^{7}+\frac{7}{2461} \cdot x^{5} z^{8}+\ldots\right] \\
& g_{6}=\frac{1}{10059033600} \cdot\left[x^{6} z^{6}+\frac{1}{2} \cdot x^{6} z^{7}+\frac{7}{52} \cdot x^{6} z^{8}+\frac{1}{39} \cdot x^{6} z^{9}+\ldots\right]
\end{aligned}
$$

all with assigned scope $x, y$ and initial monomials of the form $x^{k} z^{k}$. We keep the coefficients in front of the brackets since they will play a crucial role later on. The general formula for these and the next elements $g_{k}$ appearing in the algorithm is

$$
g_{k}=x^{k} \cdot \sum_{i=k}^{\infty} q_{i, k} \cdot z^{i}
$$

with coefficients $q_{i, k}$ given by

$$
q_{i, k}=\frac{(i-1) !}{4^{k-1} \cdot(i-k) ! \cdot(i+k-1) ! \cdot\left(\frac{1}{2}\right)^{\overline{k-1}}}
$$

where $\left(\frac{1}{2}\right)^{\overline{k-1}}$ denotes the rising factorial $\frac{1}{2}\left(\frac{1}{2}+1\right) \cdots\left(\frac{1}{2}+k-2\right)$. The expansion of $g_{k}$ results from the linear combination

$$
S\left(g_{k-1}, h\right):=-y \cdot g_{k-1}+q_{k-1, k-1} \cdot x^{k-1} z^{k-2} \cdot h
$$

of $g_{k-1}$ and $h$ given by the relation $\left(-y, x^{k-1} z^{k-2}\right)$ between their initial monomials $x^{k-1} z^{k-1}$ and $y z$, taking into account the factor $q_{k-1, k-1}$ in front of $g_{k-1}$. Then $g_{k}$ is obtained as the remainder of the division of $S\left(g_{k-1}, h\right)$ by the series $f, g, h$ and $g_{2}, \ldots, g_{k-1}$ as described in Thm. 1. All $g_{k}$ are convergent series with assigned scope $x, y$.

The vital observation here is that the coefficients $q_{k, k}$ of the initial monomials $x^{k} z^{k}$ of $g_{k}$ tend very fast to 0: more precisely, the successive quotients $q_{k, k} / q_{k+1, k+1}$ are quadratic polynomials in $k$. This convergence is caused by the rapidly decreasing coefficients $\frac{1}{k !}$ in $g=x \cdot\left(e^{z}-1\right)$ (most other transcendental series $g$ with this property would also produce counterexamples, whereas the phenomenon does not occur for algebraic power series.)

Rewrite now the series $g_{k}$ as linear combinations of the original generators $f, g, h$ of $I$,

$$
g_{k}=a_{k} \cdot f+b_{k} \cdot g+c_{k} \cdot h
$$

with uniquely defined convergent series $a_{k}, b_{k} \in \mathbb{C}\{x, y\}$ and $c_{k} \in \mathbb{C}\{x, y, z\}$. They are given by the recursions

$$
\begin{aligned}
& a_{k}=-y \cdot a_{k-1}+\frac{1}{4(2 k-3)(2 k-5)} \cdot x^{2} \cdot a_{k-2}, \\
& b_{k}=-y \cdot b_{k-1}+\frac{1}{4(2 k-3)(2 k-5)} \cdot x^{2} \cdot b_{k-2}, \\
& c_{k}=-y \cdot c_{k-1}+\frac{1}{4(2 k-3)(2 k-5)} \cdot x^{2} \cdot c_{k-2}-z^{-1} \cdot\left(a_{k-1} \cdot f+b_{k-1} \cdot g+c_{k-1} \cdot h\right),
\end{aligned}
$$

with $a_{1}=0, a_{2}=x^{2}, b_{1}=1, b_{2}=-y+\frac{1}{2} x, c_{1}=0, c_{2}=-z^{-1} \cdot x \cdot\left(1-e^{z}\right)$. The preceding formulas imply that $a_{k}, b_{k}$ are homogeneous polynomials in $x$ and $y$ of degree $k$, respectively $k-1$, while $c_{k}$ is a polynomial in $x, y, z, e^{z}, z^{-1}$ without poles. Note that in the expansions of $a_{k}, b_{k}$ and $c_{k}$ the monomials $x^{2} y^{k-2}, y^{k-1}$ and $x y^{k-2}$, respectively, appear with coefficients \pm 1 . 
The successive quotients

$$
\frac{q_{k+1, k+1}}{q_{k, k}}=\frac{1}{4 \cdot(2 k+1) \cdot(2 k-1)}
$$

of the coefficients of $g_{k}$ tend quadratically towards 0 . As

$$
\frac{q_{i, k}}{q_{k, k}}=\frac{k ! \cdot(i-1) !}{(i-k) ! \cdot(i+k-1) !} \leq 1
$$

for $i \geq k$, all coefficients $q_{i, k}$ of the series $g_{k}$ become comparatively small to $q_{k, k}$ while $k$ increases. This then implies that infinite linear combinations of the series $g_{k}$ with rapidly increasing coefficients may still produce convergent series. A typical example would be the convergent series

$$
e(x, z):=\sum_{k=2}^{\infty} \frac{1}{q_{k, k}} \cdot g_{k}(x, z) .
$$

Various other combinations of the series $g_{k}$ could be taken. By construction, the series $e$ belongs to the intersection $\widehat{I} \cap \mathbb{C}\{x, y, z\}$. We show that it does not belong to $I$. By uniqueness of the presentation, it suffices to write $e$ as a linear combination $e=a \cdot f+b \cdot g+c \cdot h$ of $f, g, h$ with divergent series $a, b, c$. Set $r_{k}=\frac{1}{q_{k, k}}$ so that $e=\sum_{k=1}^{\infty} r_{k} \cdot g_{k}$ and $a=\sum r_{k} \cdot a_{k}, b=\sum r_{k} \cdot b_{k}, c=\sum r_{k} \cdot c_{k}$ with $a_{k}$, $b_{k}$ and $c_{k}$ as defined above. As we noted earlier, the monomials $x^{2} y^{k-2}, y^{k-1}$ and $x y^{k-2}$ appear with coefficients \pm 1 in the expansions of $a_{k}, b_{k}$ and $c_{k}$, respectively. As the successive quotients $r_{k+1} / r_{k}$ tend quadratically with $k$ to infinity, it follows that the series $a, b, c$ diverge.

Remark. Here is an intuitive argument why Gabrielov's example works: The key is the replacement of the $y$-multiples $x y z^{k}$ of the monomials $x z^{k}$ of the series $g$ by $x^{2} z^{k-1}$, after multiplication of $g$ with $y$ and then applying the replacement of $y$ using $h=y z-x$. This construction corresponds to a shift by $(1,0,-1)$ of the exponents of $g$. The difference $g(x, y, z)-\frac{x}{z} \cdot g(x, y, z)$ together with the iterates of this procedure creates a sequence of series whose initial monomials have coefficients tending rapidly to 0 . This, in turn, creates the explosion of the coefficients when expressing $e$ as a linear combination of $f$, $g, h$.

\section{Outlook}

Let us conclude with a remark on how to construct further counterexamples to linear nested Artin approximation for analytic functions. The three series $f=1, g=x \cdot\left(e^{z}-1\right)$ and $h=y z-x$ proposed by Gabrielov have two key properties which make the example work: First, equating the last one to 0 , solving for $z$ (the exponent of $e^{z}$ in $g$ ), and substituting $z$ in $g$ produces $x \cdot\left(e^{\frac{x}{y}}-1\right)$, with an essential singularity at 0 with respect to $y$. This ensures that the sum in the echelon $I$ is direct, a fact which is needed to exclude non-trivial linear relations between $f, g$, and $h$. Secondly, the coefficients of $g$ tend sufficiently fast to 0 (in the example, they are $\frac{1}{k !}$ ). This allows to define a fourth convergent series $e$ as an infinite linear combination of the standard basis elements $g_{k}$ with rapidly increasing constant coefficients. As a consequence, the coefficient series $a, b, c$ in the presentation $e=a \cdot f+b \cdot g+c \cdot h$ must diverge. These are the only two properties used to produce the counterexample.

In view of this one could start off with an arbitrary $h=P(x, y) \cdot z-Q(x, y)$, with polynomials $P$ and $Q$ so that $Q / P$ has a pole at 0 , set again $f=1$ and take for $g$ any convergent power series in $z$ whose coefficients tend to 0 at least as fast as $\frac{1}{k !}$. Up to applying some simple algebraic modifications to $g$ and $h$ in order to avoid that the initial echelon in $(I)$ is finitely generated one will get again a counterexample.

Gabrielov's example and the preceding analysis of its underlying pattern is nicely contrasted by the theorem of Eisenstein and Heine about the behaviour of the coefficients of algebraic power series [Eis, Hei, DP]: 
Let $h(x) \in \mathbb{Q}[[x]]$ be a univariate algebraic power series. There exists a positive integer $m$ so that $h(m x) \in \mathbb{Z}[[x]]$ has integer coefficients. In particular, the denominators of the coefficients of $h(x)$ have only finitely many prime factors and grow at most exponentially as $m^{k}$.

This result may give a hint why there is no counterexample to Grothendieck's question for algebraic power series (a hint which is a fact by Popescu's theorem).

\section{References}

[ACH] Alonso, M.E., Castro-Jiménez, F.J., Hauser, H.: Encoding algebraic power series. Found. Comp. Math. 2017, to appear.

[Ar] Artin, M.: Algebraic Spaces. Yale Univ. Press 1971.

[Bu] Buchberger, B.: Ein algorithmisches Kriterium für die Lösbarkeit eines algebraischen Gleichungssystems (An Algorithmic Criterion for the Solvability of Algebraic Systems of Equations). Aequationes math. 3 (1970), 374-383.

[DP] Dwork, B., van der Poorten, A.: The Eisenstein constant. Duke Math. J. 65 (1992), 23-43.

[Eis] Eisenstein, G.: Über eine allgemeine Eigenschaft der Reihen-Entwicklungen aller algebraischen Funktionen. Bericht Königl. Preuss. Akad. Wiss. Berlin (1852), 441-443. Reproduced in Mathematische Gesammelte Werke, Band II, Chelsea Publishing 1975, pp. 765-767.

[Hei] Heine, E.: Theorie der Kugelfunktionen, 2nd ed., Reimer Berlin, 1878.

[Gal] Galligo, A.: A propos du Théorème de Préparation de Weierstrass. Lecture Notes in Math. 409, 543-579. Springer 1973.

[Gb1] Gabrielov, A.: Formal relations among analytic functions. Funct. Anal. Appl. 5 (1971), 318-319.

[Gb2] Gabrielov, A.: The formal relations between analytic functions. Math. USSR. Izv. 7 (1973), 1056-1088.

[GP] Greuel, G.-M., Pfister, G.: A Singular Introduction to Commutative Algebra, 2nd edition. Springer 2007.

[Gr] Grothendieck, A.: Séminaire H. Cartan 1960/1961. Familles d'espaces complexes et fondements de la géométrie analytique. Fasc. 2, Exp. 13. Secr. Math. Paris 1962.

[HM] Hauser, H., Müller, G.: A rank theorem for analytic maps between power series spaces. Publ. Math. I.H.E.S. 80 (1995), 95-115.

[Os] Osgood, W.: On functions of several complex variables, Trans. Amer. Math. Soc. 17 (1916), 1-8.

[Pa1] Pawłucki, W.: On relations among analytic functions and geometry of sub-analytic sets. Bull. Polish Acad. Sci. Math. 37 (1989), 117-125.

[Pa2] Pawłucki, W.: On Gabrielov's regularity condition for analytic mappings. Duke Math. J. 65 (1992), 299-311.

[Po1] Popescu, D.: General Néron desingularization. Nagoya Math. J. 100 (1985), 97-126.

[Po2] Popescu, D.: General Néron desingularization and approximation. Nagoya Math. J. 104 (1986), 85-115.

[Sp] Spivakovsky, M.: A new proof of D. Popescu's theorem on smoothing of ring homomorphisms. J. AMS. 12 (1999), 381-444.

[Te] Teissier, B.: Résultats récents sur l'approximation des morphismes en algèbre commutative. Sém. Bourbaki 784 (1993/94). Astérisque 227 (1995), 259-282.

M.E.A.: Dept. de Álgebra,

Universidad Complutense de Madrid, Spain mariemi@mat.ucm.es

F.J.C.-J.: Dept. de Álgebra,

Universidad de Sevilla, Spain

castro@us.es
H.H.: Faculty of Mathematics, University of Vienna, Austria herwig.hauser@univie.ac.at

C.K.: RICAM,

Austrian Academy of Sciences

christoph.koutschan@ricam.oeaw.ac.at 